\title{
Investigation of the Post-Synthetic Confinement of Fluorous Liquids Inside Mesoporous Silica Nanoparticles
}

Amani L Lee, Sang-Hyuk Lee, Huan Nguyen, Meghan Cahill, Elaine Kappel, William C.K. Pomerantz, Christy L. Haynes

\section{Supporting Information}

\section{Contents (in order of appearance)}

Description of Data Analysis

- $\quad$ Figure S1: Loading yield and efficiency of PFD PERFUMNs

- Scheme S1: Loading process to generate PERFUMNs

- Figure S2: Theoretical model of loading yield and efficiency.

- Table S1: Benchmark characterization of UMNs

- Figure S3: FITC-labeled MSNs loaded with PSLM

Description of Synthesis of FITC-APTES-MSNs

- Figure S4: Emulsion behavior between PFC and water with acidic and basic $\mathrm{pH}$

- Table S2: Experimental data of trial factors and UMN Recovery

- Table S3: Loading measurements, yields, and efficiencies of PFD PERFUMNs

- Figure S5: Design Expert statistical report for the yield.

- Figure S6: Design Expert statistical report for the efficiency.

- Figure S7: 3D models of loading yield and efficiency based on experimental trials

- Figure S8: Yields, efficiencies, and recovery of PLSM trials from optimized parameters

- Figure S9: Cryo-TEM images of PFD PERFUMNs

\section{Data Analysis}

All descriptive statistics were performed with Graphpad Prism 6.07 (GraphPad Software, La Jolla California USA, www.graphpad.com); outliers were determined by the ROUT method with a $Q$ level of $0.1 \%$. Differences in the trials were screened with a two-way ANOVA followed by a Tukey multiple comparison test.

Factor model fitting and optimization were performed with Design Expert 12 (Stat-Ease, Inc. 1300 Godward St NE \#6400, Minneapolis, MN 55413). The three factors were treated as continuously numerical variables. Models for ANOVA model fitting were determined by selecting all interaction terms with $p$-value $<0.05$. The model was then optimized by reducing the corrected Akaike information criterion (AICc), a likelihood statistic used to determine best fit models, through the removal of interaction terms. Lastly, non-significant interaction terms were added to keep hierarchy in the model (e.g., need $A$ if you have $A^{2}, A B, A C, A B C$ terms). Both models (i.e., yield, efficiency) passed the lack-of-fitness test ( $p$-value $>0.1$ ). Of note, individual replicates of yield values were above $100 \%$ during model fitting, however they could not be removed after outlier testing. These values over $100 \%$ pushed the predictive boundaries for the loading model slightly beyond $100 \%$. 


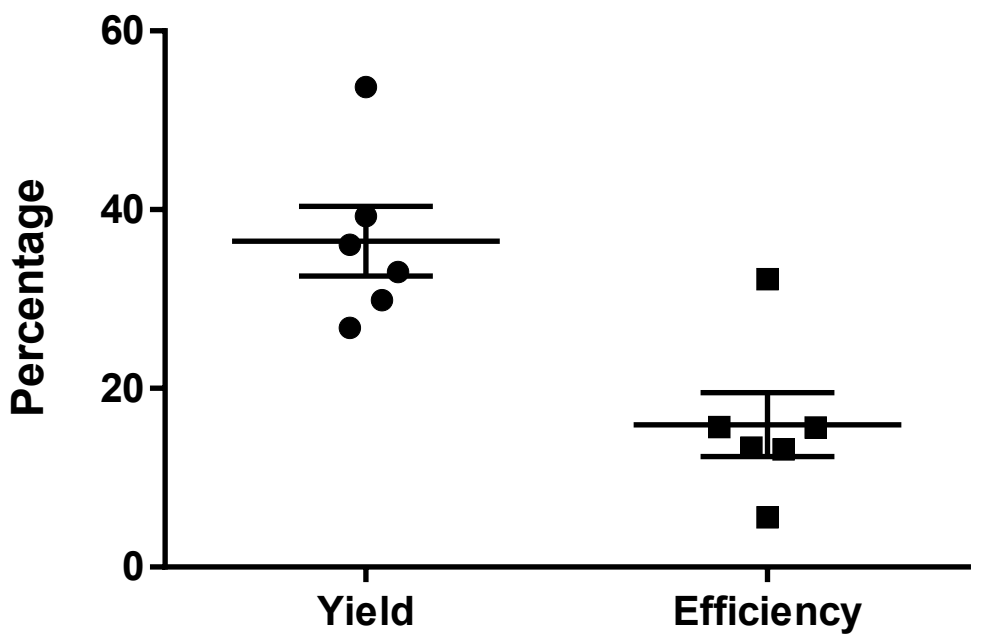

Figure S1: Loading yield and efficiency of PFD PERFUMNs from previous loading procedures reported by Lee et al. ACS Nano, 2017. ${ }^{1}$ Standard conditions were: $\mathrm{pH} \sim 7$, sonication time $=10 \mathrm{~min}, \mathrm{~V}_{R}=1$, $[\mathrm{UMN}]_{0}=3 \mathrm{mg} \mathrm{mL}^{-1}$. The mean yield and efficiency were $36.5 \pm 3.9 \%$ and $16 \pm 3.6 \%$ respectively (mean \pm SEM).

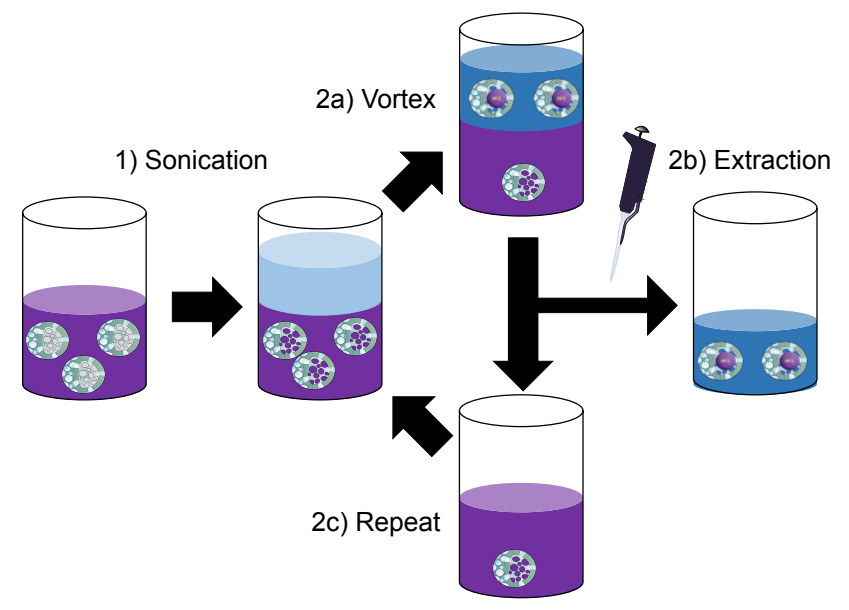

Scheme S1: Loading process to generate PERFUMNs. (1) The dried UMN nanoparticles are submerged into the neat PFC liquid and are then sonicated to fill the void space in the center of the nanoparticles. (2a) The extraction aqueous phase is added to the tube, and the two phases are vortexed for 30 seconds. (2b) The higher density fluorous phase separates from the aqueous phase, allowing facile extraction of the PERFUMNs. (2c) The process is repeated until the final volume of water reaches a desired concentration (typically $1 \mathrm{mg} \mathrm{mL}^{-1}$ ). 


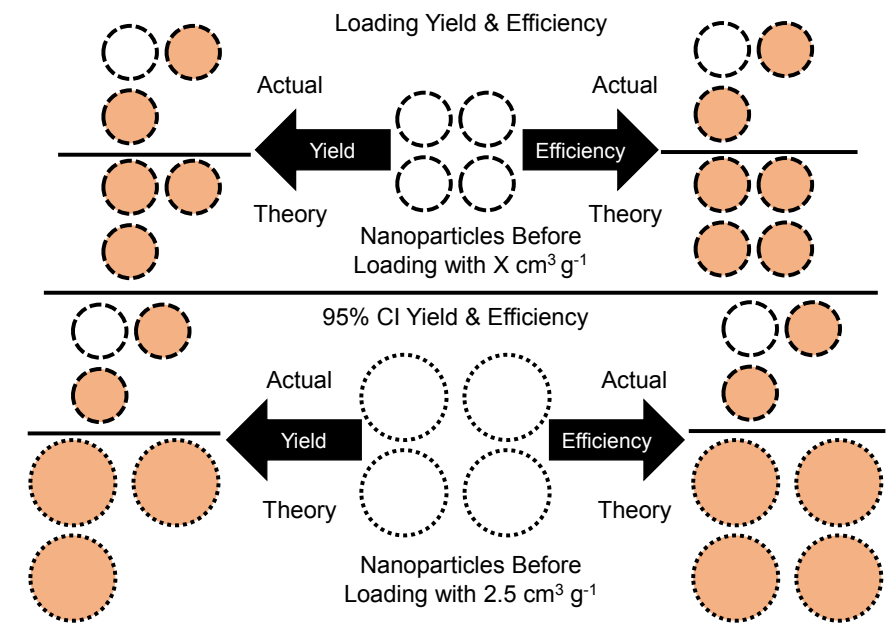

Figure S2: Theoretical model of loading yield and efficiency. The loading volume $\left(\mathrm{cm}^{3} \mathrm{~g}^{-1}\right)$ is determined by $\mathrm{N}_{2}$ physisorption measurements. The volume of the batch (the population) is assumed to translate to liquid loading capacity. The loading volume of the population translates to a subset of nanoparticles in suspension, and the total volume can be determined from knowing the mass concentration of particles in suspension. The yield accounts for the loss of loaded volume, by the loss of nanoparticle mass after extractions. The difference between the experimental and theoretical values should be due to the amount of fluorocarbon liquid that was not trapped. The efficiency additionally assumes $100 \%$ extraction of nanoparticles. The efficiency difference is attributed to loss of nanoparticles, and the loss of PFC that could have been loaded when extracted. The 95\% confidence interval $(\mathrm{Cl})$ yield \& efficiency assume the same things as the batch-specific yield and efficiency; however, it sets the theoretical loading volume to the $95 \% \mathrm{Cl}$ upper limit for UMN batches $\left(2.5 \mathrm{~cm}^{-3} \mathrm{~g}^{-1}\right)$. Essentially, it compares how the specific batch compares to the benchmark loading of all other UMN batches.

Table S1: Benchmark Characterization of Ultraporous Mesostructured Silica Nanoparticles

\begin{tabular}{l||ccc} 
Parameter & $\begin{array}{c}\text { Estimated } \\
\text { Value }\end{array}$ & $\begin{array}{c}\text { Standard } \\
\text { Deviation }\end{array}$ & $\begin{array}{c}\text { Confidence Interval } \\
(0.95)\end{array}$ \\
\hline \hline $\begin{array}{l}\text { Hydrodynamic diameter } \\
(\mathrm{nm})\end{array}$ & 142 & \pm 33 & \pm 21 \\
BJH Surface Area $\left(\mathrm{m}^{2} \mathrm{~g}^{-1}\right)$ & 532.4 & \pm 0.06 & \pm 0.04 \\
BJH Pore Volume $\left(\mathrm{cm}^{3} \mathrm{~g}^{-1}\right)$ & 1.8 & \pm 0.3 & \pm 0.7 \\
\hline \hline
\end{tabular}

Data reported previously by Lee \& Gee et al. ACS Nano $2017 .{ }^{1}$ 


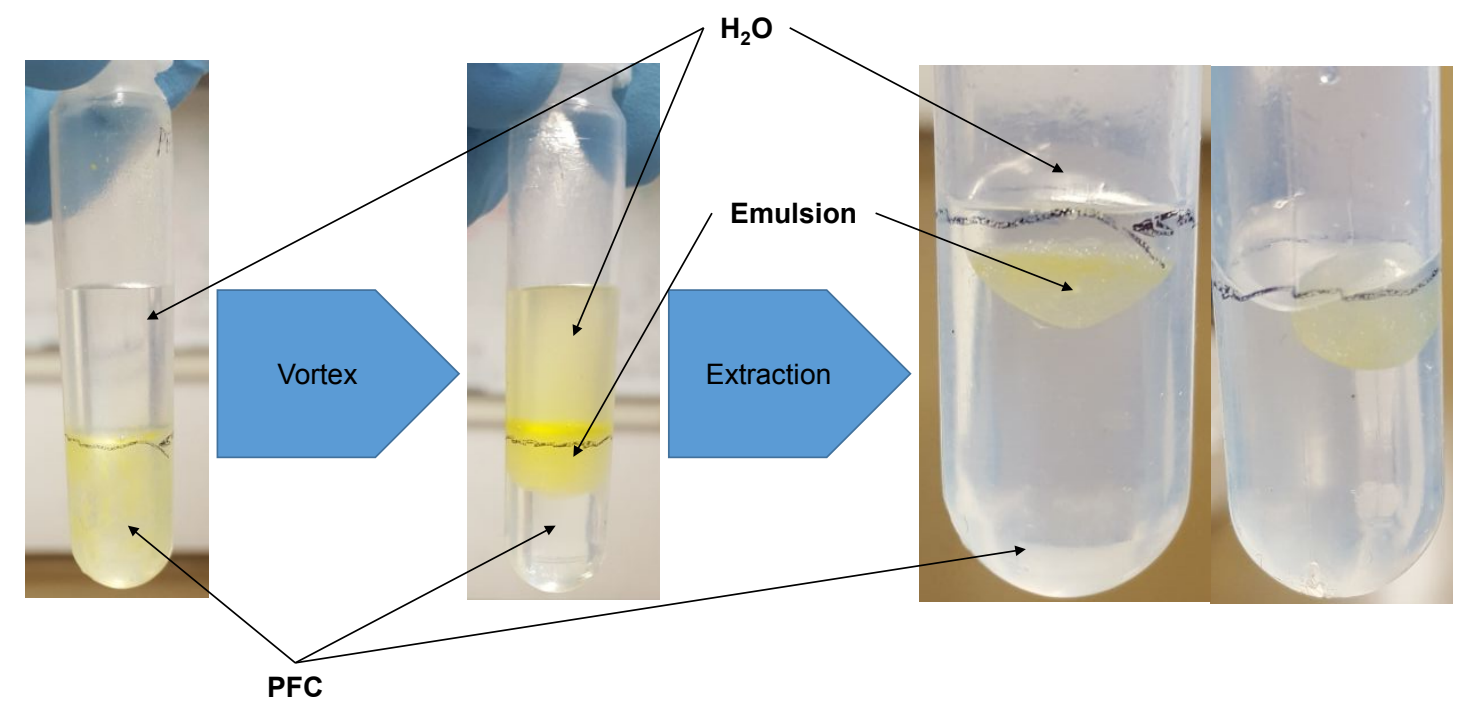

Figure S3: Tracking FITC-labeled MSN nanoparticles during the PERFUMN loading \& extraction process for ease of visualization. Nanoparticles start in the fluorous phase (black mark on tube indicates the fluorous boundary). After mixing the two layers they separate, and the FITC nanoparticles move to the aqueous phase above. After the extraction, nanoparticles are trapped in the bubble with an air-water-PFC interface.

\section{Synthesis of FITC-APTES-MSNs}

Fluorescent nanoparticles were synthesized from a previous protocol for FITCMS42@PEG/TMS-hy-c nanoparticles. ${ }^{2}$ The fluorescent dye was incorporated into the silica nanoparticles by first dissolving $5.6 \mathrm{mg}$ of fluorescein isothiocyanate (FITC) in $3 \mathrm{~mL}$ of 200 proof ethanol and $6 \mathrm{~mL}$ of (3-aminopropyl)triethyoxysilane (APTES) to conjugate FITC onto APTES (FITC-APTES). Mesoporous silica nanoparticles (MSNs) were synthesized by stirring $0.29 \mathrm{~g}$ cetyltrimethylammonium bromide (CTAB) in $150 \mathrm{~g} \mathrm{NH}_{4} \mathrm{OH}$ for 60 minutes at $50{ }^{\circ} \mathrm{C}$. After an hour $2.5 \mathrm{~mL}$ of $0.88 \mathrm{M}$ tetraethylorthosilicate and $1 \mathrm{~mL}$ of the FITC-APTES solution were added to the suspension of CTAB micelles to form FITC-APTES-MSNs. After an hour, $450 \mu \mathrm{L}$ of 2-[methoxy(polyethyleneoxy) ${ }_{9-12}$ propyl] trimethoxysilane were added, and the nanoparticle suspension was stirred for 30 minutes. The nanoparticles were washed and centrifuged several times as described in the previous protocol. 


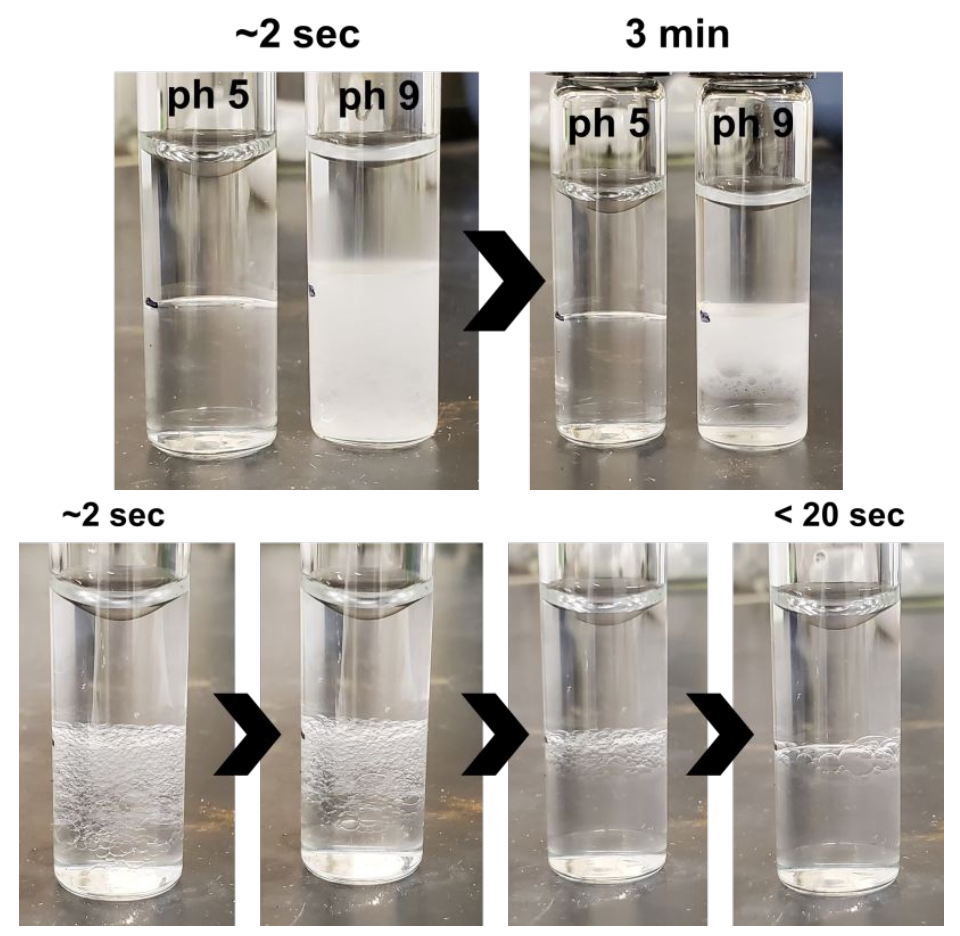

Figure S4: Emulsion formation between perfluorotripentylamine (PFTPA) \& water with $\mathrm{pH} 5$ or $\mathrm{pH}$ 9. Water was made by using dilute $(0.01 \mathrm{M})$ solutions of $\mathrm{HCl}$ or $\mathrm{NaOH}$, respectively. PFTPA was purchased from Sigma-Aldrich and the neat liquid was used directly from the bottle to cross-validate the phenomenon and reduce confounding variables from recycling used PFC-nanoparticle suspensions. This behavior was also observed with perfluorodecalin and perfluoro-15-crown-5-ether (not shown here).

Table S2: Experimental Data of Trial Factors and UMN Recovery

\begin{tabular}{|c|c|c|c|c|c|c|}
\hline Trial & $\mathrm{pH}$ & $\begin{array}{l}\text { Sonication } \\
\text { (min) }\end{array}$ & $V_{R}$ & $\begin{array}{l}{[\mathrm{UMN}]_{0}} \\
\mathrm{mg} \mathrm{mL}^{-1}\end{array}$ & $\begin{array}{l}{[\mathrm{UMN}]_{\max }} \\
\mathrm{mg} \mathrm{mL}^{-1}\end{array}$ & $\begin{array}{l}{[\mathrm{UMN}]_{\text {recovery }}} \\
\mathrm{mg} \mathrm{mL}^{-1}\end{array}$ \\
\hline 1111-1 & 4.8 & 10 & 0.8 & $\overline{c 1.0}$ & 1.0 & 1.5 \\
\hline $1111-2$ & 4.8 & 10 & 0.8 & 1.0 & 1.0 & 1.5 \\
\hline $1111-3$ & 4.8 & 10 & 0.8 & 1.0 & 1.0 & 1.3 \\
\hline $1112-1$ & 5.2 & 10 & 0.8 & 2.9 & 0.9 & 0.6 \\
\hline $1112-2$ & 5.2 & 10 & 0.8 & 3.0 & 0.9 & 0.4 \\
\hline $1112-3$ & 5.2 & 10 & 0.8 & 3.0 & 0.9 & 0.5 \\
\hline 1113-1 & 5.2 & 10 & 0.8 & 4.0 & 0.9 & 0.9 \\
\hline $1113-2$ & 5.2 & 10 & 0.8 & 4.1 & 0.9 & 0.9 \\
\hline $1113-3$ & 5.2 & 10 & 0.8 & 3.8 & 0.9 & 0.4 \\
\hline $1121-1$ & 5.4 & 10 & 1 & 1.0 & 0.6 & 0.1 \\
\hline $1121-2$ & 5.4 & 10 & 1 & 0.9 & 0.6 & 0.2 \\
\hline $1121-3$ & 5.4 & 10 & 1 & 0.8 & 0.5 & 0.3 \\
\hline $1122-1$ & 5.2 & 10 & 1 & 3.1 & 0.8 & 0.7 \\
\hline $1122-2$ & 5.2 & 10 & 1 & 2.9 & 0.8 & 0.1 \\
\hline $1122-3$ & 5.2 & 10 & 1 & 2.9 & 0.8 & 0.4 \\
\hline
\end{tabular}




\begin{tabular}{|c|c|c|c|c|c|c|}
\hline $1123-1$ & 5.2 & 10 & 1 & 4.0 & 0.9 & 0.7 \\
\hline 1123-2 & 5.2 & 10 & 1 & 3.8 & 0.8 & 0.5 \\
\hline $1123-3$ & 5.2 & 10 & 1 & 4.1 & 0.9 & 0.6 \\
\hline $1131-1$ & 4.8 & 10 & 1.2 & 1.0 & 0.5 & 0.1 \\
\hline $1131-2$ & 4.8 & 10 & 1.2 & 1.1 & 0.5 & 0.7 \\
\hline $1131-3$ & 4.8 & 10 & 1.2 & 1.1 & 0.5 & 0.0 \\
\hline $1132-1$ & 5.2 & 10 & 1.2 & 3.0 & 0.9 & 0.9 \\
\hline $1132-2$ & 5.2 & 10 & 1.2 & 3.1 & 0.9 & 0.9 \\
\hline $1132-3$ & 5.2 & 10 & 1.2 & 3.0 & 0.9 & 0.8 \\
\hline 1133-1 & 5.0 & 10 & 1.2 & 4.2 & 0.9 & 0.8 \\
\hline 1133-2 & 5.0 & 10 & 1.2 & 4.1 & 0.9 & 0.8 \\
\hline 1133-3 & 5.0 & 10 & 1.2 & 3.9 & 0.8 & 0.6 \\
\hline $1211-1$ & 5.4 & 20 & 0.8 & 1.1 & 0.9 & 0.5 \\
\hline $1211-2$ & 5.4 & 20 & 0.8 & 1.1 & 0.8 & 3.5 \\
\hline $1211-3$ & 5.4 & 20 & 0.8 & 1.2 & 0.9 & 0.4 \\
\hline $1212-1$ & 5.0 & 10 & 0.8 & 3.2 & 0.9 & 1.0 \\
\hline $1212-2$ & 5.0 & 10 & 0.8 & 3.1 & 0.9 & 0.8 \\
\hline $1212-3$ & 5.0 & 10 & 0.8 & 3.0 & 0.9 & 0.9 \\
\hline $1213-1$ & 5.0 & 20 & 0.8 & 4.0 & 0.9 & 0.8 \\
\hline $1213-2$ & 5.0 & 20 & 0.8 & 4.0 & 0.9 & 0.9 \\
\hline $1213-3$ & 5.0 & 20 & 0.8 & 4.0 & 0.9 & 0.8 \\
\hline $1221-1$ & 5.4 & 20 & 1 & 1.2 & 0.7 & 0.6 \\
\hline $1221-2$ & 5.4 & 20 & 1 & 1.1 & 0.6 & 0.2 \\
\hline $1221-3$ & 5.4 & 20 & 1 & 1.1 & 0.6 & 0.2 \\
\hline $1222-1$ & 5.0 & 20 & 1 & 3.0 & 0.8 & 0.8 \\
\hline $1222-2$ & 5.0 & 20 & 1 & 3.0 & 0.8 & 0.7 \\
\hline $1222-3$ & 5.0 & 20 & 1 & 3.1 & 0.8 & 0.7 \\
\hline $1223-1$ & 5.0 & 20 & 1 & 4.1 & 0.9 & 0.5 \\
\hline $1223-2$ & 5.0 & 20 & 1 & 4.0 & 0.9 & 0.5 \\
\hline $1223-3$ & 5.0 & 20 & 1 & 4.2 & 0.9 & 0.7 \\
\hline $1231-1$ & 4.8 & 20 & 1.2 & 1.1 & 0.5 & 0.1 \\
\hline $1231-2$ & 4.8 & 20 & 1.2 & 1.1 & 0.5 & 0.1 \\
\hline $1231-3$ & 4.8 & 20 & 1.2 & 1.1 & 0.6 & 0.1 \\
\hline $1232-1$ & 5.2 & 20 & 1.2 & 3.0 & 0.9 & 0.3 \\
\hline $1232-2$ & 5.2 & 20 & 1.2 & 3.0 & 0.9 & 0.2 \\
\hline $1232-3$ & 5.2 & 20 & 1.2 & 2.8 & 0.8 & 0.1 \\
\hline $1233-1$ & 5.1 & 20 & 1.2 & 4.1 & 0.9 & 0.8 \\
\hline $1233-2$ & 5.1 & 20 & 1.2 & 4.1 & 0.9 & 0.8 \\
\hline $1233-3$ & 5.1 & 20 & 1.2 & 4.3 & 0.9 & 0.6 \\
\hline $1311-1$ & 4.8 & 30 & 0.8 & 1.0 & 0.8 & 0.1 \\
\hline $1311-2$ & 4.8 & 30 & 0.8 & 1.1 & 0.9 & 0.1 \\
\hline $1311-3$ & 4.8 & 30 & 0.8 & 1.0 & 0.8 & 0.0 \\
\hline $1312-1$ & 5.1 & 30 & 0.8 & 3.0 & 0.9 & 0.9 \\
\hline $1312-2$ & 5.1 & 30 & 0.8 & 3.0 & 0.9 & 0.5 \\
\hline $1312-3$ & 5.1 & 30 & 0.8 & 3.1 & 0.9 & 1.0 \\
\hline $1313-1$ & 5.1 & 30 & 0.8 & 4.0 & 0.9 & 0.9 \\
\hline $1313-2$ & 5.1 & 30 & 0.8 & 4.1 & 0.9 & 0.9 \\
\hline $1313-3$ & 5.1 & 30 & 0.8 & 4.0 & 0.9 & 0.6 \\
\hline $1321-1$ & 4.8 & 30 & 1 & 1.0 & 0.6 & 0.6 \\
\hline $1321-2$ & 4.8 & 30 & 1 & 1.1 & 0.7 & 0.1 \\
\hline
\end{tabular}




\begin{tabular}{l||llllll}
$1321-3$ & 4.8 & 30 & 1 & 1.1 & 0.6 & 0.0 \\
\cline { 2 - 7 } $1322-1$ & 5.0 & 30 & 1 & 3.1 & 0.8 & 0.6 \\
$1322-2$ & 5.0 & 30 & 1 & 3.1 & 0.8 & 0.7 \\
$1322-3$ & 5.0 & 30 & 1 & 3.1 & 0.8 & 0.6 \\
\cline { 2 - 7 } $1323-1$ & 5.0 & 30 & 1 & 4.0 & 0.9 & 0.9 \\
$1323-2$ & 5.0 & 30 & 1 & 4.1 & 0.9 & 0.9 \\
$1323-3$ & 5.0 & 30 & 1 & 4.0 & 0.9 & 1.0 \\
\cline { 2 - 7 } $1331-1$ & 4.8 & 30 & 1.2 & 1.1 & 0.5 & 0.0 \\
$1331-2$ & 4.8 & 30 & 1.2 & 1.1 & 0.5 & 0.1 \\
$1331-3$ & 4.8 & 30 & 1.2 & 1.1 & 0.5 & 0.0 \\
\cline { 2 - 7 } $1332-1$ & 5.0 & 30 & 1.2 & 3.0 & 0.9 & 0.6 \\
$1332-2$ & 5.0 & 30 & 1.2 & 3.1 & 0.9 & 0.5 \\
$1332-3$ & 5.0 & 30 & 1.2 & 3.0 & 0.9 & 0.6 \\
\cline { 2 - 7 } $1333-1$ & 5.0 & 30 & 1.2 & 4.1 & 0.9 & 1.0 \\
$1333-2$ & 5.0 & 30 & 1.2 & 4.0 & 0.9 & 0.8 \\
$1333-3$ & 5.0 & 30 & 1.2 & 4.3 & 0.9 & 1.0 \\
\hline
\end{tabular}

Table S3: Loading Measurements, Yields, and Efficiencies of PFD PERFUMNs

\begin{tabular}{c||cccccc}
\multicolumn{1}{c||}{ Trial } & $\boldsymbol{V}_{\boldsymbol{a v g}}^{\boldsymbol{S}}$ & $\begin{array}{c}\text { [PFD }]_{\text {NMR }} \\
(\mathrm{mM})\end{array}$ & $\begin{array}{c}\boldsymbol{\gamma} \\
(\%)\end{array}$ & $\begin{array}{c}\boldsymbol{\gamma}^{*} \\
(\%)\end{array}$ & $\begin{array}{c}\boldsymbol{\gamma}_{\mathbf{0 . 9 5} \boldsymbol{C I}} \\
(\%)\end{array}$ & $\begin{array}{c}\boldsymbol{\gamma}_{\mathbf{0 . 9 5}}^{*} \boldsymbol{C I} \\
(\%)\end{array}$ \\
\hline \hline $1111-1$ & 1.45 & 0.45 & 5.3 & 7.9 & 3.1 & 4.6 \\
$1111-2$ & 1.45 & 0.2 & 2.4 & 3.6 & 1.4 & 2.1 \\
$1111-3$ & 1.45 & 0.26 & 3.5 & 4.5 & 2.0 & 2.6 \\
\cline { 2 - 6 } $1112-1$ & 1.98 & 2.68 & 54.2 & 40.4 & 43.0 & 27.2 \\
$1112-2$ & 1.98 & 2.28 & 70.3 & 33.7 & 55.7 & 23.2 \\
$1112-3$ & 1.98 & 1.84 & 49.8 & 26.9 & 39.5 & 18.8 \\
\cline { 2 - 6 } $1113-1$ & 1.42 & 2.11 & 40.2 & 42.1 & 22.8 & 21.5 \\
$1113-2$ & 1.42 & 1.88 & 38.9 & 36.2 & 22.1 & 19.1 \\
$1113-3$ & 1.42 & 1.02 & 46.8 & 21.1 & 26.6 & 10.4 \\
\cline { 2 - 6 } $1121-1$ & 2.2 & 0.47 & 65.6 & 9.0 & 57.6 & 4.8 \\
$1121-2$ & 2.2 & 0.51 & 29.3 & 10.3 & 25.7 & 5.1 \\
$1121-3$ & 2.2 & 0.47 & 21.9 & 10.9 & 19.2 & 4.8 \\
\cline { 2 - 6 } $1122-1$ & 1.47 & 1.64 & 42.3 & 34.4 & 25.0 & 16.6 \\
$1122-2$ & 1.47 & 0.5 & 68.5 & 11.4 & 40.4 & 5.0 \\
$1122-3$ & 1.47 & 0.7 & 28.9 & 16.0 & 17.0 & 7.1 \\
\cline { 2 - 6 } $1123-1$ & 1.47 & 2.03 & 47.2 & 40.2 & 27.8 & 20.6 \\
$1123-2$ & 1.47 & 1.38 & 45.2 & 28.6 & 26.7 & 14.0 \\
$1123-3$ & 1.47 & 1.71 & 45.9 & 33.4 & 27.1 & 17.4 \\
\cline { 2 - 6 } $1131-1$ & 2.25 & 1.28 & 108.6 & 28.4 & 97.6 & 13.0 \\
$1131-2$ & 2.25 & 1.17 & 20.4 & 25.5 & 18.4 & 11.9 \\
$1131-3$ & 2.25 & 1.35 & 919.3 & 28.9 & 826.0 & 13.8 \\
\cline { 2 - 6 } $1132-1$ & 1.75 & 2.64 & 40.8 & 44.7 & 28.5 & 26.9 \\
$1132-2$ & 1.75 & 3.12 & 50.9 & 51.0 & 35.6 & 31.7 \\
$1132-3$ & 1.75 & 2.97 & 51.9 & 50.3 & 36.3 & 30.2 \\
\cline { 2 - 6 } $1133-1$ & 1.75 & 2.93 & 51.2 & 48.5 & 35.8 & 29.8 \\
$1133-2$ & 1.75 & 2.93 & 56.2 & 48.4 & 39.3 & 29.8
\end{tabular}




\begin{tabular}{|c|c|c|c|c|c|c|}
\hline 1133-3 & 1.75 & 2.48 & 57.7 & 43.4 & 40.3 & 25.2 \\
\hline $1211-1$ & 2.2 & 2.37 & 53.1 & 31.9 & 46.7 & 24.1 \\
\hline $1211-2$ & 2.2 & 1.72 & 5.8 & 23.7 & 5.1 & 17.5 \\
\hline $1211-3$ & 2.2 & 1.65 & 52.1 & 21.0 & 45.8 & 16.8 \\
\hline $1212-1$ & 1.75 & 3.44 & 51.9 & 53.9 & 36.2 & 35.0 \\
\hline $1212-2$ & 1.75 & 3.06 & 55.8 & 48.5 & 39.0 & 31.2 \\
\hline $1212-3$ & 1.75 & 3.26 & 51.4 & 54.0 & 35.9 & 33.2 \\
\hline $1213-1$ & 1.52 & 2.51 & 51.9 & 45.6 & 31.6 & 25.5 \\
\hline $1213-2$ & 1.52 & 2.38 & 45.5 & 43.3 & 27.7 & 24.2 \\
\hline $1213-3$ & 1.52 & 2.39 & 47.4 & 43.4 & 28.8 & 24.3 \\
\hline $1221-1$ & 2.2 & 1.52 & 32.1 & 25.2 & 28.2 & 15.5 \\
\hline $1221-2$ & 2.2 & 1.79 & 112.9 & 32.3 & 99.2 & 18.2 \\
\hline $1221-3$ & 2.2 & 1.34 & 103.2 & 24.2 & 90.6 & 13.6 \\
\hline $1222-1$ & 1.52 & 1.79 & 37.5 & 38.5 & 22.8 & 18.2 \\
\hline $1222-2$ & 1.52 & 1.87 & 43.2 & 40.1 & 26.3 & 19.0 \\
\hline $1222-3$ & 1.52 & 1.76 & 42.0 & 36.8 & 25.5 & 17.9 \\
\hline $1223-1$ & 1.52 & 2.24 & 83.2 & 42.6 & 50.6 & 22.8 \\
\hline $1223-2$ & 1.52 & 1.99 & 66.5 & 38.7 & 40.4 & 20.2 \\
\hline $1223-3$ & 1.52 & 2.18 & 53.5 & 39.7 & 32.5 & 22.2 \\
\hline $1231-1$ & 1.54 & 0.2 & 67.5 & 6.2 & 41.5 & 2.1 \\
\hline $1231-2$ & 1.54 & 0.32 & 106.1 & 10.2 & 65.2 & 3.3 \\
\hline $1231-3$ & 1.54 & 0.35 & 43.1 & 10.3 & 26.5 & 3.5 \\
\hline $1232-1$ & 1.98 & 1.33 & 57.0 & 19.7 & 45.2 & 13.6 \\
\hline $1232-2$ & 1.98 & 1.45 & 123.7 & 21.3 & 98.1 & 14.7 \\
\hline $1232-3$ & 1.98 & 1.87 & 410.6 & 29.2 & 325.5 & 19.0 \\
\hline $1233-1$ & 1.55 & 2.09 & 45.7 & 38.9 & 28.3 & 21.2 \\
\hline 1233-2 & 1.55 & 2.02 & 41.4 & 38.1 & 25.7 & 20.6 \\
\hline $1233-3$ & 1.55 & 2.64 & 72.3 & 47.6 & 44.8 & 26.9 \\
\hline $1311-1$ & 1.25 & 1 & 245.6 & 25.9 & 122.6 & 10.2 \\
\hline $1311-2$ & 1.25 & 0.86 & 210.9 & 20.7 & 105.2 & 8.8 \\
\hline $1311-3$ & 1.25 & 0.69 & 840.7 & 17.5 & 419.5 & 7.0 \\
\hline $1312-1$ & 1.55 & 2.58 & 47.0 & 48.6 & 29.1 & 26.2 \\
\hline $1312-2$ & 1.55 & 2.87 & 91.2 & 53.5 & 56.6 & 29.2 \\
\hline $1312-3$ & 1.55 & 2.59 & 43.1 & 47.1 & 26.8 & 26.3 \\
\hline $1313-1$ & 1.55 & 2.6 & 48.8 & 46.9 & 30.3 & 26.5 \\
\hline $1313-2$ & 1.55 & 2.75 & 53.0 & 48.9 & 32.9 & 27.9 \\
\hline $1313-3$ & 1.55 & 2.55 & 65.1 & 45.9 & 40.4 & 25.9 \\
\hline $1321-1$ & 1.54 & 0.45 & 13.1 & 12.1 & 8.0 & 4.6 \\
\hline $1321-2$ & 1.54 & 0.57 & 141.6 & 14.1 & 87.0 & 5.8 \\
\hline $1321-3$ & 1.54 & 0.28 & 276.4 & 7.2 & 169.9 & 2.8 \\
\hline $1322-1$ & 1.24 & 2.57 & 92.9 & 64.2 & 46.2 & 26.2 \\
\hline $1322-2$ & 1.24 & 2.78 & 76.7 & 70.2 & 38.1 & 28.3 \\
\hline $1322-3$ & 1.24 & 2.77 & 93.0 & 69.0 & 46.3 & 28.1 \\
\hline $1323-1$ & 1.24 & 2.66 & 54.0 & 61.8 & 26.9 & 27.1 \\
\hline $1323-2$ & 1.24 & 2.3 & 54.9 & 53.5 & 27.3 & 23.4 \\
\hline $1323-3$ & 1.24 & 2.6 & 54.9 & 61.0 & 27.3 & 26.4 \\
\hline $1331-1$ & 1.54 & 0.25 & 121.8 & 7.8 & 74.9 & 2.5 \\
\hline $1331-2$ & 1.54 & 0.06 & 12.8 & 2.0 & 7.9 & 0.7 \\
\hline $1331-3$ & 1.54 & 0.31 & 311.1 & 9.6 & 191.3 & 3.2 \\
\hline $1332-1$ & 1.24 & 1.87 & 64.6 & 44.0 & 32.2 & 19.0 \\
\hline
\end{tabular}




\begin{tabular}{l||llllll}
$1332-2$ & 1.24 & 2.02 & 77.5 & 45.9 & 38.5 & 20.6 \\
$1332-3$ & 1.24 & 1.78 & 65.1 & 41.3 & 32.4 & 18.1 \\
\cline { 2 - 7 } $1333-1$ & 1.55 & 2.63 & 43.8 & 49.5 & 27.2 & 26.7 \\
$1333-2$ & 1.55 & 2.48 & 51.9 & 47.8 & 32.2 & 25.2 \\
$1333-3$ & 1.55 & 2.99 & 49.1 & 54.0 & 30.5 & 30.5 \\
\hline
\end{tabular}

Columns from left to right: Trials, loading capacity (determined from BJH pore volume), loading yield, loading efficiency, normalized loading yield (@ $0.95 \mathrm{Cl}$ ), normalized loading efficiency (@ $0.95 \mathrm{Cl}$ ).

\begin{tabular}{|c|c|c|c|c|c|c|c|}
\hline Source & $\begin{array}{l}\text { Sum of } \\
\text { Squares }\end{array}$ & df & $\begin{array}{l}\text { Mean } \\
\text { Square }\end{array}$ & F-value & p-value & \multicolumn{2}{|c|}{ Relative Impact of Factors } \\
\hline Model & 204.78 & 11 & 18.62 & 13.88 & $<0.0001$ & Sqrt(Yield) & $=$ \\
\hline $\begin{array}{l}\text { A- } \\
\text { Sonication }\end{array}$ & 15.98 & 1 & 15.98 & 11.92 & 0.0010 & +7.92 & \\
\hline $\begin{array}{l}\text { B-Vol } \\
\text { Ratio }\end{array}$ & 1.57 & 1 & 1.57 & 1.17 & 0.2829 & $\begin{array}{r}+1.10 \\
+0.3541\end{array}$ & $\begin{array}{l}\text { A: Sonication Time (min) } \\
\text { B: Vol Ratio }\end{array}$ \\
\hline $\mathrm{C}$-[UMN]o & 22.91 & 1 & 22.91 & 17.08 & 0.0001 & +0.3041 & D. $v^{R}$, vol katio \\
\hline$A B$ & 0.0746 & 1 & 0.0746 & 0.0557 & 0.8143 & -0.7529 & C: [UMN]。 \\
\hline $\mathrm{AC}$ & 47.37 & 1 & 47.37 & 35.32 & $<0.0001$ & +0.0921 & $A B$ \\
\hline $\mathrm{BC}$ & 2.69 & 1 & 2.69 & 2.00 & 0.1622 & -1.43 & $A C$ \\
\hline $\mathrm{C}^{2}$ & 0.0146 & 1 & 0.0146 & 0.0109 & 0.9173 & -0.3218 & $\mathrm{BC}$ \\
\hline$A B C$ & 25.47 & 1 & 25.47 & 18.99 & $<0.0001$ & +0.0338 & $C^{2}$ \\
\hline$A C^{2}$ & 2.24 & 1 & 2.24 & 1.67 & 0.2014 & & 4 \\
\hline $\mathrm{BC}^{2}$ & 0.0665 & 1 & 0.0665 & 0.0496 & 0.8245 & +1.32 & $\mathrm{ABC}$ \\
\hline$A B C^{2}$ & 17.53 & 1 & 17.53 & 13.07 & 0.0006 & +0.5140 & $A C^{2}$ \\
\hline Residual & 79.12 & 59 & 1.34 & & & +0.0892 & $\mathrm{BC}^{2}$ \\
\hline Lack of Fit & 26.47 & 15 & 1.76 & 1.47 & 0.1569 & -1.77 & $A B C^{2}$ \\
\hline Pure Error & 52.65 & 44 & 1.20 & & & & \\
\hline Cor Total & 283.90 & 70 & & & & & \\
\hline
\end{tabular}

Figure S5: Design Expert statistical report for the yield. (Left) The ANOVA test for the model of loading factors that explains the variation in the yield. (Right) Relative impact of the factors; the larger the absolute value of the coefficient, the more the yield is expected to change. The factors are coded by letters, i.e., $A=S o n i c a t i o n$ time $(\min ), B=$ Volume Ratio $\left(V_{R}\right), C=[U M N]_{0}$. 


\begin{tabular}{|c|c|c|c|c|c|c|}
\hline Source & $\begin{array}{l}\text { Sum of } \\
\text { Squares }\end{array}$ & df & Mean Square & F-value & p-value & Relative Impact of Factors \\
\hline Model & 196.80 & 22 & 8.95 & 75.67 & $<0.0001$ & Sqrt(Efficiency) $=$ \\
\hline A-Sonication & 20.00 & 1 & 20.00 & 169.15 & $<0.0001$ & +5.78 \\
\hline B-Vol Ratio & 9.43 & 1 & 9.43 & 79.76 & $<0.0001$ & $+2.34 \mathrm{~A}=$ Sonication Time $(\mathrm{min})$ \\
\hline C-[UMN]o & 2.31 & 1 & 2.31 & 19.57 & $<0.0001$ & $-1.45 B=$ Vol Ratio $\left(V_{R}\right)$ \\
\hline$A B$ & 12.18 & 1 & 12.18 & 103.04 & $<0.0001$ & $+0.6076 \mathrm{C}=[\mathrm{UMN}]_{0}$ \\
\hline$A C$ & 2.18 & 1 & 2.18 & 18.42 & $<0.0001$ & $-0.6109 A B$ \\
\hline $\mathrm{BC}$ & 2.61 & 1 & 2.61 & 22.06 & $<0.0001$ & $+0.4260 \mathrm{AC}$ \\
\hline$A^{2}$ & 0.4439 & 1 & 0.4439 & 3.75 & 0.0578 & $+0.4661 \mathrm{BC}$ \\
\hline$A B C$ & 6.46 & 1 & 6.46 & 54.62 & $<0.0001$ & $-0.3595 A^{2}$ \\
\hline$A^{2} B$ & 8.14 & 1 & 8.14 & 68.88 & $<0.0001$ & $+0.5395 \mathrm{ABC}$ \\
\hline$A^{2} C$ & 5.40 & 1 & 5.40 & 45.72 & $<0.0001$ & $+1.66 A^{2} B$ \\
\hline $\mathrm{AB}^{2}$ & 11.83 & 1 & 11.83 & 100.07 & $<0.0001$ & $+1.15 \mathrm{~A}^{2} \mathrm{C}$ \\
\hline $\mathrm{AC}^{2}$ & 9.00 & 1 & 9.00 & 76.13 & $<0.0001$ & $-2.13 A B^{2}$ \\
\hline $\mathrm{B}^{2} \mathrm{C}$ & 1.74 & 1 & 1.74 & 14.73 & 0.0003 & $-1.84 A C^{2}$ \\
\hline $\mathrm{BC}^{2}$ & 2.39 & 1 & 2.39 & 20.19 & $<0.0001$ & $+0.6423 \mathrm{~B}^{2} \mathrm{C}$ \\
\hline$A^{2} B^{2}$ & 3.92 & 1 & 3.92 & 33.18 & $<0.0001$ & $+0.8851 \mathrm{BC}^{2}$ \\
\hline $\mathrm{A}^{2} \mathrm{BC}$ & 2.16 & 1 & 2.16 & 18.30 & $<0.0001$ & $+0.7921 \mathrm{~A}^{2} \mathrm{~B}^{2}$ \\
\hline$A^{2} C^{2}$ & 1.02 & 1 & 1.02 & 8.66 & 0.0047 & $-0.5303 A^{2} B C$ \\
\hline $\mathrm{AB}^{2} \mathrm{C}$ & 0.7891 & 1 & 0.7891 & 6.67 & 0.0125 & $-0.4277 \mathrm{~A}^{2} \mathrm{C}^{2}$ \\
\hline $\mathrm{B}^{2} \mathrm{C}^{2}$ & 2.09 & 1 & 2.09 & 17.69 & $<0.0001$ & $-0.3202 A^{2} C$ \\
\hline$A^{2} B^{2} C$ & 2.21 & 1 & 2.21 & 18.71 & $<0.0001$ & $-0.5157 \mathrm{~B}^{2} \mathrm{C}^{2}$ \\
\hline$A^{2} B C^{2}$ & 1.35 & 1 & 1.35 & 11.46 & 0.0013 & $-0.8974 A^{2} B^{2} C$ \\
\hline$A B^{2} C^{2}$ & 5.62 & 1 & 5.62 & 47.53 & $<0.0001$ & $-0.8226 A^{2} B C^{2}$ \\
\hline Residual & 6.50 & 55 & 0.1182 & & & $+1.75 \mathrm{AB}^{2} \mathrm{C}^{2}$ \\
\hline Lack of Fit & 0.2551 & 4 & 0.0638 & 0.5207 & 0.7209 & $+1.15 \mathrm{AB}^{2} \mathrm{C}^{2}$ \\
\hline Pure Error & 6.25 & 51 & 0.1225 & & & \\
\hline Cor Total & 203.30 & 77 & & & & \\
\hline
\end{tabular}

Figure S6: Design Expert statistical report for the efficiency. (Left) The ANOVA test for the model of loading factors that explain the variation in the yield. (Right) Relative impact of the factors; the larger the absolute value of the coefficient, the more the yield is expected to change. The factors are coded by letters, i.e., A = Sonication time $(\mathrm{min})$, $\mathrm{B}=$ Volume Ratio $\left(\mathrm{V}_{\mathrm{R}}\right), \mathrm{C}=[\mathrm{UMN}]_{\mathrm{o}}$. 


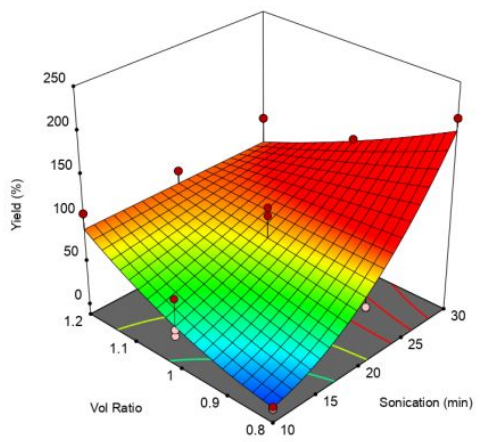

$[\mathrm{UMN}]_{0}=1 \mathrm{mg} \mathrm{mL}^{-1}$

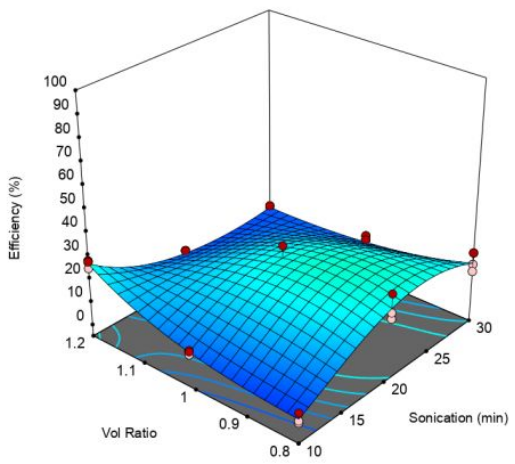

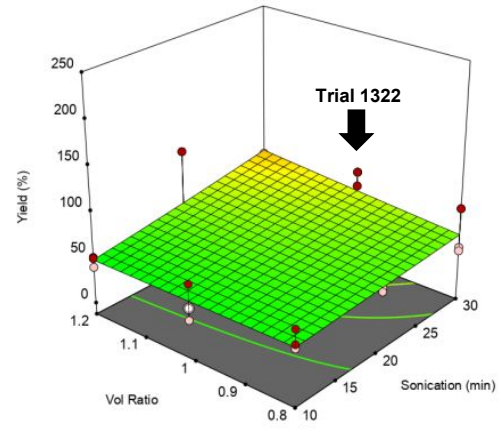

$[\mathrm{UMN}]_{\mathrm{o}}=3 \mathrm{mg} \mathrm{mL}^{-1}$

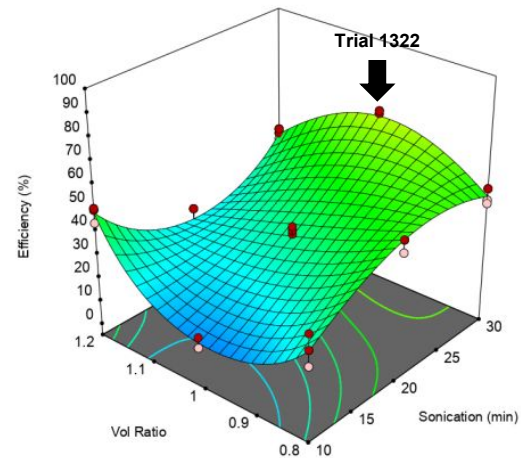

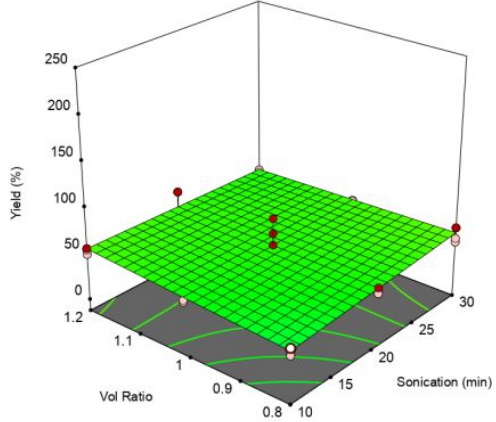

$[\mathrm{UMN}]_{\mathrm{o}}=4 \mathrm{mg} \mathrm{mL}^{-1}$

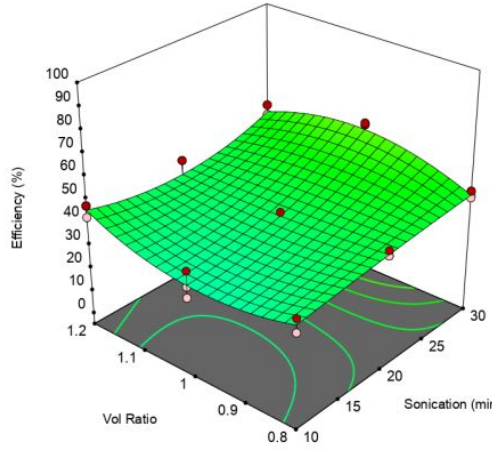

Figure S7: 3D models of loading yield and efficiency based on experimental trials. The colored surface is based on predictions of the different factors based on the fitted model from analysis. Trial 1322 is marked by an arrow. The red and white dots represent experimental trials, where red dots are above the surface and white are below the surface. Sonication and the volume ratio $\left(V_{R}\right)$ make up the $x$ - and $y$-axes; the loading yield (top row) or efficiency (bottom row) are on the z-axis. The graphs are separated into 3 columns based on the initial nanoparticle concentration [UMN]。 from 1-4 mg mL-1. The graphs suggest that increasing the sonication time and $V_{R}$ will increase the yield exponentially, at $1 \mathrm{mg} \mathrm{mL}^{-1}$. Efficiency is estimated to be most optimal at $3 \mathrm{mg} \mathrm{mL}^{-1}$ with a long sonication time and a $V_{R}=1$. 


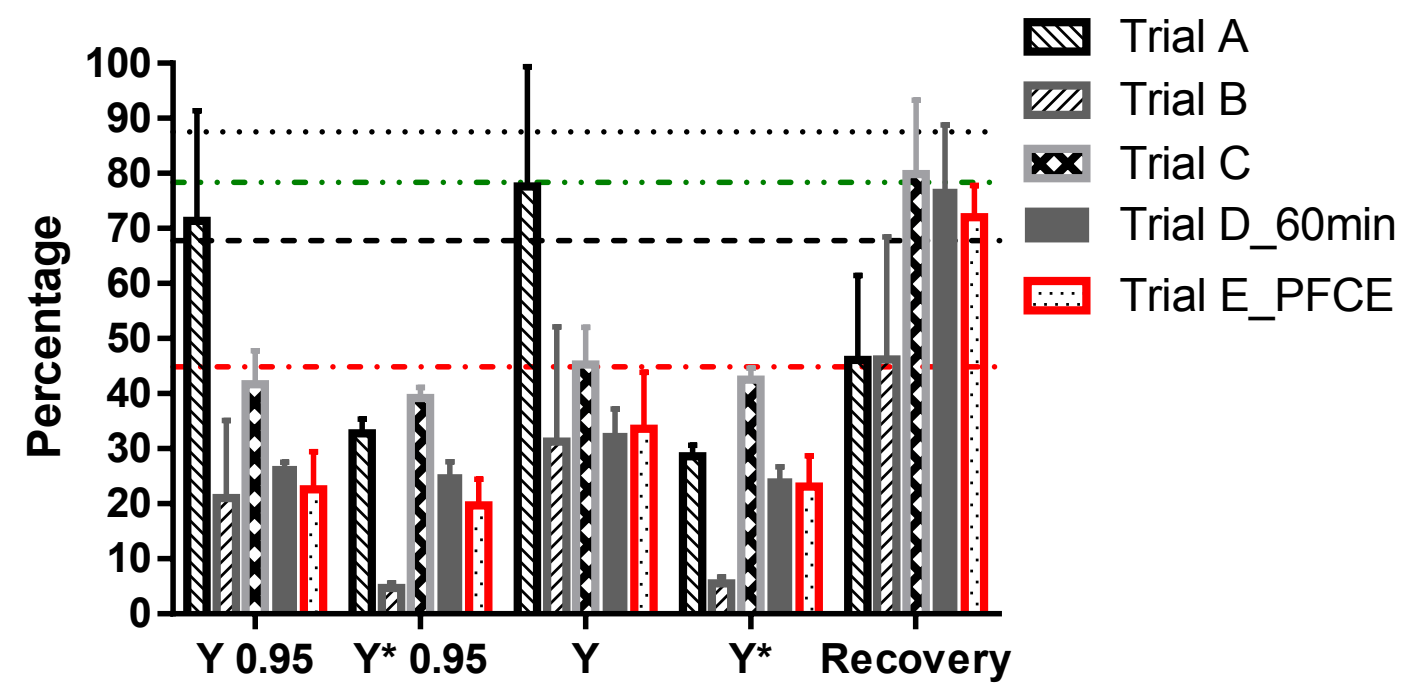

Figure S8: Yields, efficiencies, and nanoparticle recovery of PLSM trials from optimized parameters. $Y$ and $Y^{*}$ correspond to yield and efficiency, respectively. The " 0.95 " notation corresponds to normalized values. Recovery is the experimental concentration $\div$ theoretical max concentration after extraction. Each trial was done in triplicate $(n=3)$. Trial A-C were PFD PERFUMNs loaded with model optimized parameters. Trial D was PFD PERFUMNs with 60 minute sonication time, all other parameters were similar to Trial A-C. Trial E was PFCE PERFUMNs with same parameters as Trials A-C. Lines correspond to previous benchmarks: (dot) average yield of Trial 1322; (dash) average efficiency of Trial 1322; (dash-dot-dash, red) recovery of PFCE PERFUMNs in Lee et al.; and (Dash-dot-dot-dash, green) the percent recovery from Trial 1322. 

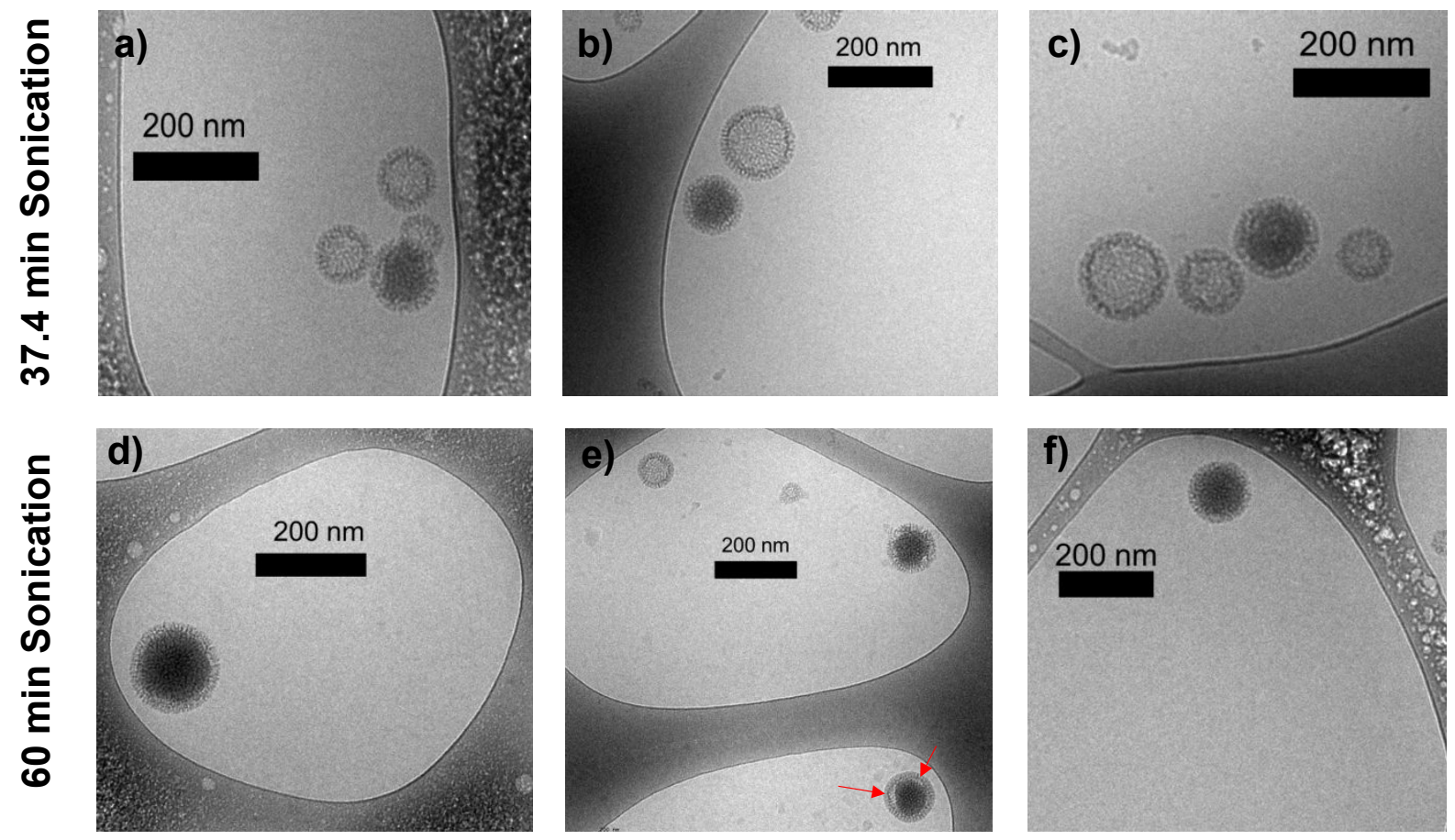

Figure S9: Cryo-TEM images of PFD PERFUMNs that were sonicated for 37.4 min (top) or 60 min (bottom), $1.1 V_{R}$, $2.6 \mathrm{mg} \mathrm{mL}^{-1}$ [UMN], and pH 5 conditions. (a-c) Nanoparticles after sonicated for 37.4 min; many of the nanoparticles are unfilled, however, those that were filled did not show prominent nano-sized bubbles in the PFC phase. Small gaps are markedly near the boundary of PFC and the silica shell. (d-f) Nanoparticles sonicated for 60 min; crescentshaped gaps are less prominent but not eradicated. Red arrows image "e" show void space within the core of the nanoparticle causing and oblong shape in the PFC droplet.

\section{References}

1. Lee, A. L.; Gee, C. T., et al. Oxygen Sensing with Perfluorocarbon-Loaded Ultraporous Mesostructured Silica Nanoparticles. ACS Nano 2017, 11, 5623-5632.

2. Lin, Y. S.; Abadeer, N.; Hurley, K. R.; Haynes, C. L. Ultrastable, Redispersible, Small, and Highly Organomodified Mesoporous Silica Nanotherapeutics. J. Am. Chem. Soc. 2011, 133, 20444-20457. 